\title{
Discourse and Mental Health. Voice, Inequality and Resistance in Medical Settings
}

JUAN EdUARDo Bonnin. 2019.

LONDON: ROUTLEDGE. 192 PÁGINAS.

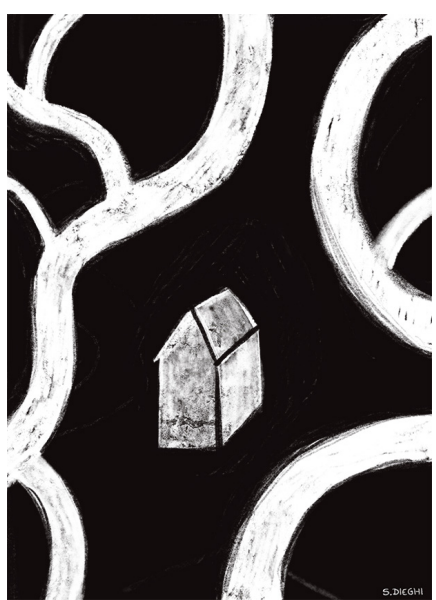

- Antonio M. Bañón Hernández

Universidad de Almería, España

Trabajo recibido el 27 de mayo de 2019 y aprobado el 18 de junio de 2019.

El análisis del discurso aplicado a temas sanitarios ha suscitado en las últimas décadas un gran interés por parte de investigadores dedicados, de alguna manera, al estudio de la comunicación. Igual ocurre si nos centramos específicamente en el ámbito de la salud mental. De hecho, bastaría con hacer un recorrido, por ejemplo, por los contenidos de la revista Journal of Mental Health para comprobarlo. Tres han sido las dimensiones principalmente abordadas: el discurso como demostración de salud o de enfermedad, el discurso como herramienta generadora de salud o de enfermedad y el discurso como manifestación de una determinada construcción social de la salud y de la enfermedad. El libro que ahora reseñamos estaría situado preferentemente en la tercera dimensión. Ahora bien, hemos de decir que, con un valor añadido: la importancia que tiene la construcción de lo que significa estar sano o estar enfermo, así como de la enfermedad misma en el acceso a los servicios sanitarios (lo que nos conduciría a la segunda dimensión: el discurso como herramienta generadora de salud a través de la psicoterapia). Sin duda, las investigaciones orientadas no únicamente a identificar objetos social y discursivamente relevantes y a describirlos a partir de las herramientas que nos proporciona el análisis del discurso, sino también a aplicar el trabajo realizado a realidades complejas, como la salud y la enfermedad, con la intención de mejorar la calidad de vida de las personas en situación de desventaja, han de ser siempre bienvenidas. Si, además, esas investigaciones se hacen con la calidad de este libro escrito por Juan Eduardo Bonnin, la alegría es aún mayor.

Bonnin, que defiende, con razón, tanto la vinculación directa entre lo crítico y lo discursivo como la idea según la cual cada proyecto de investigación es el resultado de un debate entre diferentes actores, se ocupa de las relaciones entre discurso y desigualdad en el ámbito de la salud mental. Ha hecho un trabajo de campo de cinco años en un hospital público localizado en una barriada de Buenos Aires con una amplia presencia de vecinos inmigrados. Se trata de conocer cómo las prácticas discursivas inciden y expresan la diversidad y la desigualdad en el acceso a los servicios de salud mental. Con frecuencia, propone una aproximación diferente a la comunicación médico-paciente en salud mental, concediendo la importancia merecida a la diversidad cultural, social y económica. En cierto sentido, vale decir que el análisis de este tipo de interacciones implica la adecuada consideración del contexto.

El libro se estructura en siete capítulos, a los que antecede una introducción y sigue un epílogo. El primero de esos capítulos se titula "Voice, Singularity and Emergency". Es muy interesante observar cómo puede llegar a sorprender en contextos de salud mental algo tan sencillo como lo siguiente: un marco monolingüe en relación a la población y una representación monoglósica del lenguaje no siempre encaja con la realidad; de hecho, muchas veces no encaja porque los actos de comunicación reflejan tanto la regularidad como la posibilidad de romper con ella. Por otra parte, defiende Bonnin, es esencial atender, en la caracterización de los participantes en un evento discursivo, a sus biografías individuales, así como a sus sentimientos y a sus emociones. A ello habrá que añadir el concepto "emergency". Puesto que el discurso no es algo meramente preexsitente a los hablantes, sino que la realidad comunicativa es polifónica, heteroglósica y multidimensional, en movimiento constante, el autor propone explorar el "emergent discourse". En varias ocasiones, Bonnin recuerda que hay que pensar en formas de colaboración entre el análisis del discurso y la sociolingüística para conocer las consecuencias de la desigualdad lingüística en el acceso a los ámbitos de atención sanitaria.

"Psychoanalysis in public hospitals" es el título del segundo capítulo. El subtítulo es el siguiente: "Context as a discursive 
problem". Y es verdad que, como decíamos antes, el contexto es un concepto clave, que usamos constantemente, pero no siempre con tino. De hecho, en ocasiones se observan investigaciones sobre la interacción médico-paciente que olvidan indicar algo tan esencial como es el que ese intercambio comunicativo se produzca en centros sanitarios públicos, privados o mixtos (como ocurre en Argentina). Por lo demás, recuerda Bonnin, siguiendo a van Dijk, hemos de conceder el protagonismo merecido a los modelos mentales de contexto que pueden diferir de un hablante a otro, incluso si ambos comparten un mismo espacio y un mismo tiempo.

Los pacientes que acuden a los hospitales públicos no suelen tener acceso a otro tipo de atención sanitaria. Y los pacientes necesitados de cuidados en salud mental pasan, además, desde hace algún tiempo, por unas "entrevistas de admisión”. Es un microgénero, pues, con especial trascendencia, ya que, según sea el comportamiento (también discursivo) de la persona que allí acude y su ajuste a los criterios institucionales, habrá posibilidad o no de ser admitido en el servicio. Pensemos que son hospitales que, en muchas ocasiones, como ocurre con el utilizado por el autor para su investigación, están saturados por una alta demanda a la que no siempre se puede dar respuesta con los medios existentes. Bonnin ofrece en este capítulo datos muy interesantes sobre el comienzo de los servicios de salud mental, sobre los estudios de psicología o sobre la tradición argentina en el uso del psicoanálisis. La construcción del espacio en un nivel público y también privado, así como la definición de la situación (identificando roles y actividades) completan este capítulo a través del estudio de fragmentos específicos de interacción en los que se da espacio a cuestiones tales como la mirada o las formas de tratamiento. No es extraño que haya desajustes en la construcción o en la definición entre los pacientes y los profesionales.

El capítulo 3 se titula "Invisible landscapes. Diversity and the semiosis of space”. En él, Juan Eduardo Bonnin analiza los “silent discourses" en el ámbito de la salud pública. La invisibilización de la diversidad lingüística o la privatización de la comunicación pública son dos aportaciones destacadas para todos los que estamos interesados en el discurso en entornos sanitarios. En verdad, hay distintos grados de visibilidad o de invisibilidad. El lector disfrutará de un cuidadoso análisis de los carteles, las notas manuscritas o la señalética del hospital, y de cómo tales elementos reflejan realidades estables o limitadas en el tiempo, así como presencia de actores y luchas ideológicas o profesionales. No menos importantes son las reflexiones sobre el contraste entre lo que es invisibilizado desde el punto de vista de la escritura, pero que tiene visibilidad en el ámbito oral (lenguas minoritarias, por ejemplo).

"Diagnosis and treatment. Sequencing and exclusion”, el capítulo 4, parte de la acertada idea según la cual el diagnóstico en la atención mental ha de ser abordado como un proceso, en el que no siempre coinciden quienes optan por una mirada desde el psicoanálisis o quienes defienden la mirada psiquiátrica. Además, es cierto que suele haber discrepancias entre pacientes y profesionales sanitarios a la hora de interpretar lo que ha de entenderse por "entrevista de admisión". Para los segundos es una manera de filtrar; para los primeros puede ser, de hecho, el comienzo de una actividad clínica. Hay datos que despertarán el interés, sin duda, de los lectores. Bonnin recuerda, por ejemplo, que, durante 2008, el $43 \%$ de los pacientes fue diagnosticado de Desorden de Comportamiento Disfuncional, y, sin embargo, en 2009 , únicamente el $1 \%$ recibió ese mismo diagnóstico. Cambios tan sobresalientes pueden obedecer a cuestiones no siempre claras y, en todo caso, tienen repercusiones tanto sobre el tratamiento de las personas que acuden a este hospital, como sobre el proceso comunicativo mismo. Sumemos a todo ello el que hay debates profesionales que pueden enfrentar a psicoterapeutas y a psiquiatras, que repercuten sobre el proceso de diagnóstico, pero de los que no son conocedores los pacientes.

El quinto capítulo se titula "Resisting exclusion. Pacients" tactics of misunderstanding bureaucratic discourse". El autor analiza las técnicas de expansión de las respuestas ante las preguntas realizadas por los profesionales durante la entrevista inicial. Así, los pacientes, por poner un caso, pueden adelantar información burocrática aunque no haya sido aún solicitada. Es una manera, como otras que describe cuidadosamente Bonnin, de gestionar el tiempo a favor de lo que se considera posiblemente más importante desde el punto de vista médico: la enfermedad y su posible solución o atenuación, para lo que es necesario, lógicamente, poder acceder a los servicios.

El sexto capítulo se ocupa de un tema muy tratado por la comunicación en contextos sanitarios: la acomodación entre pacientes y profesionales, que intenta aliviar la distancia y la asimetría existentes por lo normal entre unos y otros. Se trata, de alguna manera, de "hablar con la voz de los otros". No en vano, el título del capítulo es el siguiente: "Speaking with the other's voice. An attempt to closet he gap”. La aparición de expresiones técnicas es una de las fórmulas utilizadas por los pacientes y la incorporación de expresiones cronolectales o de procesos fonéticos identificativos de clases sociales estigmatizadas está presente en las intervenciones de los profesionales. Pero también son utilizados elementos léxicos propios de etnias o religiones asociadas, en ocasiones, a personas migradas de otros países hacia Argentina. Como dice Bonnin, en un momento del capítulo, el deseo de acomodación y de aproximación no siempre resulta exitoso, hasta el punto de que puede enfatizar incluso la distancia entre los interlocutores.

El último capítulo, “Discourse and activism. Dissent, protest and resistance", aborda estos tres tipos de -valdría 
decir- discurso reivindicativo que, por cierto, tienen un gran protagonismo en la vida púbica argentina y que forman parte del debate colectivo sobre temas de gran relevancia social, política o económica. Es el caso de la Ley Nacional de Salud Mental, el tema que trata Bonnin en este capítulo. El material discursivo se sale de alguna manera del utilizado en los capítulos anteriores, pero merecerá la pena su cuidadosa lectura por las incisivas reflexiones que encontrará el lector sobre cuestiones tan importantes como la desinstitucionalización.
En definitiva, estamos, como se advierte en el epílogo, ante un libro que puede ser considerado un viaje, un recorrido por una realidad diversa y compleja. Puede haber otros, pero, sin duda, se trata de una excelente contribución al análisis del discurso aplicado a la salud y a la enfermedad. Y es, además, una investigación que puede ayudar a mejorar los procesos comunicativos relacionados con la salud mental no sólo en el hospital estudiado, y no sólo en Argentina. 